удк 621.039.7

В. А. Канченко, А. А. Кучмагра,

О. С. Молчанов, Г. И. Одинокин, Н. Л. Чепур

Институт проблем безопасности АЭС НАН Украины

\section{Численный анализ} источников

\section{ионизирующего излучения камер деления}

Проведен анализ радиационной опасности источников ионизирующего излучения, применяемых в камерах деления типа КНТЗ1 и КНТ31-1, где в качестве материала радиатора используется уран, обогащенный до $90 \%$ изотопом ${ }^{235} \mathrm{U}$, с содержанием около $1 \%$ примесей ${ }^{234} U$. Рассчитаны суммарная и удельная активность, а также мощность эквивалентной дозы на расстоянии 0,1 м от поверхности источников ионизирующего излучения.

Ключевы е слов а: источник ионизирующего излучения

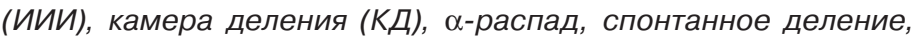
мощность эквивалентной дозы (МЭД).

В. Я. Канченко, О. А. Кучмагра, О. С. Молчанов, Г. І. Одінокін, М. Л. Чепур

Кількісний аналіз джерел іонізуючого випромінювання камер поділу

Проведено аналіз радіаційної небезпеки джерел іонізуючого випромінювання, застосовуваних у камерах поділу типу КНT31 і КНТ31-1, де матеріалом радіатора є уран, збагачений до $90 \%$ ізотопом ${ }^{235} \mathrm{U}$, з вмістом близько $1 \%$ домішок ${ }^{234} \mathrm{U}$. Розраховано сумарну й питому активність, а також потужність еквівалентної дози на відстані 0,1 м від поверхні джерел іонізуючого випромінювання.

Ключов і слов а: джерело іонізуючого випромінювання

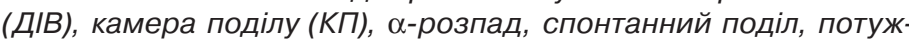
ність еквівалентної дози (ПЕД).

(C) В. А. Канченко, А. А. Кучмагра, О. С. Молчанов, Г. И. Одинокин, Н. Л. Чепур, 2011
$\mathrm{B}$ ИПБ АЭС НАНУ разработана установка оперативного контроля глубины выгорания ядерного топлива. В этой установке использованы камеры деления (КД) типа КНТ31 и КНТ31-1. К таким системам предъявляются особые требования и накладываются дополнительные условия эксплуатации, а именно:

1. В соответствии с [1] в Государственном регистре необходимо производить регистрацию ИИИ, которые одновременно отвечают следующим условиям:

- суммарная активность источника превышает $10^{4}$ Бк;

- удельная активность источника превышает 10 Бк/г;

- период полураспада радионуклида составляет более 30 дней;

- мощность эквивалентной дозы на расстоянии 0,1 м от поверхности источника превышает 1 мкЗв/ч.

2. Необходимо производить учет и контроль ядерных материалов в Государственной системе [2] в случае, когда в чувствительных элементах контрольно-измерительных приборов содержание ИИИ превышает несколько граммов.

3. Правила ведения учета и контроля ядерных материалов [3] указывают на необходимость их исполнения при использовании урана в контрольно-измерительных приборах в количестве 12 и более в одном приборе.

Обоснование необходимости регистрации (либо ее отсутствия), учета и контроля систем, имеющих в своем составе ИИИ, требует проведения тщательного анализа создаваемых ими радиационных полей, что непосредственно связано с обеспечением радиационной безопасности. Решению этих вопросов применительно к указанным камерам деления посвящена эта статья. Использованные в настоящей статье методы, приемы и подходы определения параметров различных видов ионизирующих излучений могут представлять интерес для эксплуатирующих подобные системы специалистов и использоваться ими для решения подобных задач.

Таким образом, целью настоящей работы является анализ влияния и степень воздействия ионизирующего излучения, создаваемого ИИИ указанных камер деления, на окружающую среду и обслуживающий персонал, а также соответствия (или не соответствия) параметров рассматриваемого ионизирующего излучения указанным требованиям и условиям эксплуатации их источников.

Ионизируюшее воздействие. В импульсных КД, к которым относятся КД КНТ31 и КНТ31-1, в качестве материала радиатора используется уран, обогащенный до $90 \%$ изотопом ${ }^{235} \mathrm{U}$. В таком уране обычно содержится около $1 \%$ примесей ${ }^{234} \mathrm{U}, \alpha$-активность которого гораздо больше, чем у ${ }^{235} \mathrm{U}$ [4]. Похожие данные приводятся и в [5], где показано, что в качестве материала радиатора применяется уран высокого обогащения (93\% $\left.{ }^{235} U+1 \%{ }^{234} U\right)$ или особо высокого обогащения $\left(99,9 \%{ }^{235} \mathrm{U}\right.$, менее $\left.0,03 \%{ }^{234} \mathrm{U}\right)$. Однако ввиду чрезвычайно высокой стоимости последний применяется в случае крайней необходимости снижения $\alpha$-фона ${ }^{234} U$ до уровня фона ${ }^{235} \mathrm{U}$ (в частности, в КД КНТ14 и КНТ15-1) [5], но не в рассматриваемых КНТ31 и КНТ31-1).

Ионизирующее воздействие КД на окружающую среду и обслуживающий персонал возможно в результате $\alpha$-распада урана радиатора и его спонтанного деления. В последнем случае ионизация происходит как за счет кинетической энергии осколков деления, так и в результате их дальнейшего распада (нейтронное и $\beta$-излучения). Рассмотрим все виды излучения, создаваемые в результате распада материала радиатора КД КНТ31 и КНТ31-1. 
Альфа-излучение возникает при $\alpha$-распаде урана (все изотопы урана $\alpha$-активны). Определим пробеги $\alpha$-частиц. Пробег $R$, см, $\alpha$-частицы в воздухе приближенно можно определить по формуле [6]

$$
R=0,318 T_{\alpha}^{3 / 2},
$$

где $T_{\alpha}-$ кинетическая энергия $\alpha$-частицы, МэВ.

Для подавляющего большинства $\alpha$-активных ядер $4 \mathrm{MэB} \leq T_{\alpha} \leq 9$ МэВ, среднее значение $T_{\alpha} \approx 6$ МэВ [6] (например, при распаде ${ }^{238} \mathrm{U} T_{\alpha}=4,2$ МэВ [7]). Таким образом, среднее значение пробега $\alpha$-частицы в воздухе в соответствии с формулой (1)

$$
R=0,318 \cdot 6^{3 / 2} \approx 4,7 \mathrm{~cm} .
$$

Для определения пробега $\alpha$-частицы в линейном размере в материале корпуса КД, выполненного из нержавеющей стали, воспользуемся данными, приведенными в [8] по пробегу в единицах плотности $\alpha$-частиц в железе: $R=10,73$ мг/см ${ }^{2} п р и ~ T T_{\alpha} \approx 6$ МэВ. С учетом того, что плотность железа равна $7,85 \cdot 10^{3} \mathrm{Mr} / \mathrm{cm}^{3}$,

$$
R_{\alpha}=10,73 /\left(7,85 \cdot 10^{3}\right) \mathrm{cm}=1,34 \cdot 10^{-3} \mathrm{~cm},
$$

что гораздо меньше толщины корпуса КД (около 0,6 мм).

Аналогично получим пробеги $\beta$-частиц, которые приближенно можно вычислять по эмпирической формуле (для $T_{\beta}>0,8$ МэВ) [6]

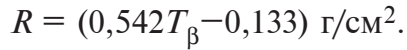

Энергия $\beta$-излучения радионуклидов не превышает 3 МэВ [9]. Согласно формуле (2),

$$
R_{\beta}=(0,542 \cdot 3-0,133) / 7,85=0,19 \text { см. }
$$

Хотя это значение и превышает толщину корпуса КД, $\beta$-излучение от КД не представляет радиационной опасности, так как потоки $\beta$-частиц в основном опасны при контактном и внутреннем облучении организма [10], тем более, что значения этих потоков не только малы сами по себе в силу крайне низкой активности по $\beta$-излучению, но и ослаблены экранирующим действием корпуса КД, о чем свидетельствует соизмеримость полученного выше значения свободного пробега электронов и толщины корпуса КД.

Излучение антинейтрино, сопровождающее $\beta$-распад, мы не рассматриваем, поскольку оно практически не взаимодействует с веществом.

Рассмотрим создаваемое осколками спонтанного деления ${ }^{235} \mathrm{U}$ нейтронное излучение при его прохождении через корпус КД, используя данные, приведенные в [10].

Слой половинного ослабления нейтронов определяется по формуле

$$
\Delta_{1 / 2}=0,693 / \Sigma,
$$

где $\Sigma=N_{a}\left(\sigma_{s}+\sigma_{a}\right)-$ полное макроскопическое сечение поглотителя; $N_{a}=\rho N_{a} / A-$ количество атомов по-

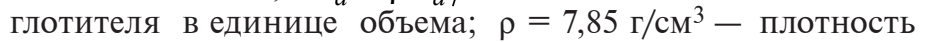
поглотителя (материала корпуса КД, в качестве которого принимаем железо); $N_{A}=6,025 \cdot 10^{23}\left(\Gamma^{\cdot}\right.$ моль) ${ }^{-1}-$ число Авогадро; $A=66$ - атомное массовое число железа;

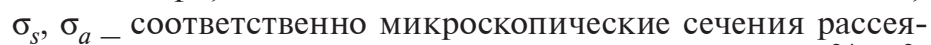
ния и поглощения (для железа $\sigma_{s}=11$ Барн $=11 \cdot 10^{-24} \mathrm{~cm}^{2}$, $\sigma_{a}=2,53$ Барн $=2,53 \cdot 10^{-24} \mathrm{~cm}^{2}[8]$.

Подставив приведенные данные в формулу (3), получим $\Delta_{1 / 2}=0,7$ см.

Таким образом, в пользу радиационной безопасности нейтронного излучения от КД служат те же соображения, что и при $\beta$-излучении (существенное его ослабление материалом корпуса КД и крайне низкая активность материала радиатора по нейтронному излучению).

Ионизирующие свойства осколков деления не представляют радиационной опасности, так как согласно [6] даже в воздухе при нормальном давлении длина пробега легкого осколка составляет 25 мм, тяжелого - 19 мм, а в фотоэмульсии, плотность которой намного меньше плотности материала корпуса КД, соответственно 13,4 и 10,4 мкм. Очевидно, что при таких значениях пробега осколков деления они, как и $\alpha$-частицы, не покидают объема КД и их ионизирующее воздействие на окружающую среду и обслуживающий персонал практически отсутствует.

Суммарная и удельная активность ИИИ. Хотя материалом радиатора является смесь изотопов урана, обогащенного на $90 \%$ по ${ }^{235} \mathrm{U}$, будем использовать консервативный подход, считая весь материал радиатора состоящим из ${ }^{235} \mathrm{U}$, имеющего наибольшую гамма-постоянную, равную $\Gamma=0,71 \mathrm{P} \cdot \mathrm{cm}^{2} /(ч \cdot \mathrm{мКи)} \mathrm{[9].}$

Активность радионуклида определяется как число его ядерных превращений в единицу времени:

$$
A=\left(N_{0}-N\right) / t=N_{0}\left(1-\exp \left\{-0,693 t / T_{1 / 2}\right\}\right) / t
$$

где $N_{0}$ - начальное число ядер радионуклида; $T_{1 / 2}=0,693=$ $=\ln 2-$ его период полураспада.

Будем рассматривать только $\alpha$-распад ${ }^{235} \mathrm{U} \quad\left(T_{1 / 2}=\right.$ $=6,85 \cdot 10^{8}$ лет [9]), поскольку его период полураспада почти на 9 порядков меньше этого параметра по спонтанному делению.

Если $A$ измерять в беккерелях, то $t=1 \mathrm{c}$, и тогда, в силу огромного значения $T_{1 / 2}=6,85 \cdot 10^{8}$ лет $=2,16 \cdot 10^{16}$ с, получаем $\exp \left\{-0,693 t / T_{1 / 2}\right\} \approx 1-0,693 t / T_{1 / 2}$. Отсюда

$$
A=0,693 N_{0} / T_{1 / 2} \text { Бк, }
$$

где величина $T_{1 / 2}$ берется в секундах.

Определим $N_{0}$ для КД КНТ31 и КНТ31-1:

$$
N_{0}=m /\left[A\left({ }^{235} \mathrm{U}\right) \cdot 1,66 \cdot 10^{-24}\right],
$$

где $m-$ масса материала радиатора, г; $A\left({ }^{235} \mathrm{U}\right)=235-$ массовое число ${ }^{235} \mathrm{U} ; 1,66 \cdot 10^{-24}$ - атомная единица массы, г.

Площадь радиатора КД КНТ31 и КД КНТ31-1 составляет $500 \mathrm{~cm}^{2}$ [4], [5], поверхностная плотность материала радиатора в обеих КД - 1 мг/см². Таким образом, для обеих КД масса ИИИ $m_{\text {Иии }}=0,5$ г; тогда начальное число ядер радионуклида

$$
N_{0}=0,5 /\left(235 \cdot 1,66 \cdot 10^{-24}\right)=1,28 \cdot 10^{21},
$$

суммарная активность

$$
A=0,693 \cdot 1,28 \cdot 10^{21} /\left(2,16 \cdot 10^{16}\right)=4,1 \cdot 10^{4} \mathrm{БK},
$$

откуда удельная активность для обеих КД составляет $8,2 \cdot 10^{4}$ БК/г.

Оценим активности материала радиатора по $\beta$ - и нейтронному излучениям (соответственно $A_{\beta}$ и $A_{n}$ ), возникающим в процессе спонтанного деления $235 \mathrm{U}$. Учитывая количество $\beta$-распадов $\left(n_{\beta}\right)$ и выход нейтронов на акт деления (v) и используя уравнение (4), получим

$$
A_{\beta} / A_{\alpha}=n_{\beta} T_{\alpha} / T_{\text {сп }} ; A_{n} / A_{\alpha}=v T_{\alpha} / T_{\text {сп }},
$$

где $T_{\alpha}=6,85 \cdot 10^{8}$ лет, $T_{\text {сп }}=1,8 \cdot 10^{17}$ лет - периоды полураспада соответственно по $\alpha$ - и спонтанному делениям. 


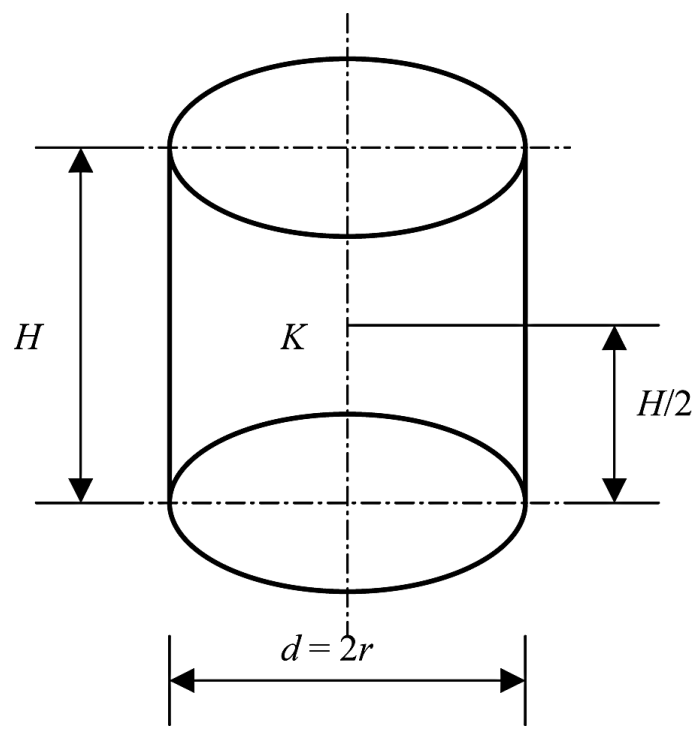

Рис. 1. ИИИ в виде цилиндра с поверхностной боковой активностью

Количество $\beta$-распадов определяется количеством звеньев в цепочке спонтанного деления ${ }^{235} \mathrm{U}: n_{\beta}=3 \div 4$ [6], а выход нейтронов в среднем (v) составляет 2,5 [6]. Подставив эти значения в (5), находим, что активности по $\beta$ - и нейтронному излучениям на 8 порядков меньше активности по $\alpha$-распаду и их можно не учитывать.

Таким образом, образуемые в рассматриваемых КД $\alpha$-излучение и все виды излучений, возникающих в результате спонтанного деления (включая и осколки деления), практически не оказывают ионизирующего воздействия на окружающую среду и обслуживающий персонал. Однако суммарная и удельная активность материала радиатора по $\alpha$-распаду, его период полураспада превышают уровни, установленные в [1].

Мощность эквивалентной дозы. Определим с учетом геометрии радиатора мощность эквивалентной дозы, создаваемой КД, использовав значения мощности воздушной кермы $\dot{K}$, которые для воздуха практически совпадают с мощностью поглощенной дозы (далее - дозы) $\dot{D}$ [9].

В КД КНТ31 материал радиатора нанесен на коаксиально расположенные цилиндры высотой (длиной чувствительной части) $H=20$ см и наружными диаметрами $d_{1}=1 \mathrm{~cm}, d_{2}=1,8 \mathrm{~cm}, d_{3}=2,6$ см и $d_{4}=3,2 \mathrm{cм} \mathrm{[4].}$

В [9] приведена формула для расчета мощности дозы ИИИ, имеющего форму цилиндра с равномерно распределенной по боковой поверхности активностью, в точках, расположенных на его оси (рис. 1).

Рассчитаем мощность дозы в геометрическом центре цилиндра (точка $K$ на рис. 1$)$, где ее значение максимально. В этом случае

$$
\dot{D}=4 \pi A_{s} \Gamma_{\delta} \operatorname{arctg}(H / d),
$$

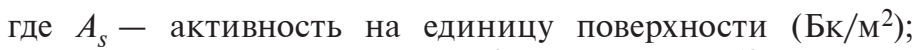
$\Gamma_{\delta}$ - керма-постоянная, аГр $\mathrm{м}^{2} /(\mathrm{c} \cdot Б \mathrm{Б})\left(\mathrm{a}=10^{-18}\right)$.

Очевидно, что мощность дозы в данной точке от разнесенных в пространстве ИИИ определяется как суперпозиция мощностей доз в данной точке от каждого источника. Исходя из этого, получим

$$
\dot{D}=4 \pi A_{s} \Gamma_{\delta} \sum_{i=1}^{4} \operatorname{arctg}(H / d) .
$$

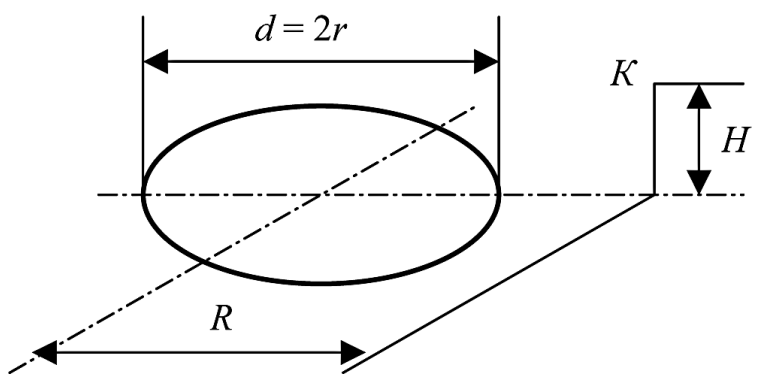

Рис. 2. ИИИ в виде круглого диска

Учитывая, что активность материала радиатора $4,1 \cdot 10^{4} \mathrm{БK}$, площадь покрытия (радиатора) $500 \mathrm{~cm}^{2}=0,05 \mathrm{M}^{2}$, находим

$$
A_{s}=4,1 \cdot 10^{4} / 0,05=8,2 \cdot 10^{5} \mathrm{БK} / \mathrm{M}^{2} .
$$

Для ${ }^{235} \mathrm{U} \Gamma_{\delta}=4,653$ aГр·м²/(c·Бк) [9]. Подставив численные значения в формулу (6), получим

$$
\dot{D}=2,8 \cdot 10^{8} \mathrm{a} \Gamma \mathrm{p} / \mathrm{c}=10^{-6} \Gamma \mathrm{p} / \text { ч }
$$

Учитывая, что $\alpha$-распад сопровождается практически только $\gamma$-излучением (не считая $\alpha$-частиц), для которого радиационный взвешивающий фактор равен единице, получим значение мощности эквивалентной дозы (МЭД)

$$
\dot{W}=\dot{D}=10^{-6} \text { Зв } / \text { ч }=1 \text { мкЗв } / \text { ч. }
$$

Как видим, в точке максимальной МЭД ее значение не превышает уровня, указанного в [1]. Очевидно, что на расстоянии 10 см от поверхности КД значение МЭД будет гораздо меньше данного уровня.

Обратимся к рассмотрению КД КНТ31-1, в которой материал радиатора нанесен на параллельно расположенные диски с диаметром $d=2,8$ см и длиной чувствительной части $L=17,6$ см [5]. Определим количество дисков $(n)$ и расстояние между чувствительными покрытиями соседних дисков $(l)$, считая расстояния между соседними дисками одинаковыми и учитывая, что площадь радиатоpa $S=500 \mathrm{~cm}^{2}$ :

$$
n=\left[S /\left(\pi r^{2}\right)\right]=\left[500 /\left(3,14 \cdot 1,4^{2}\right)\right]=[81,24]=81 \quad([X]-\text { це- }
$$
лая часть числа $X)$;

$l=17,6 /(81-1)=0,22$ см (при межэлектродном зазоре 1,6 мм).

Определим значение МЭД в плоскости среднего диска на расстоянии 10 см от его края (это точка максимального значения МЭД на расстоянии 10 см от края ИИИ):

$$
\dot{W}=\dot{W}_{0}+2 \sum_{i=1}^{40} \dot{W}_{i}
$$

где $\dot{W}_{0}-$ МЭД, создаваемая излучением от среднего диска; $\dot{W}_{i}-$ МЭД, создаваемая от $i$-го диска $(i=1 . .40)$; коэффициент 2 при сумме учитывает симметричность расположения дисков относительно среднего (по 40 дисков по обе стороны).

Используем формулу мощности воздушной кермы для диска, равномерно покрытого тонким слоем активности (рис. 2):

$\dot{K}=\pi A_{S} \Gamma_{\delta} \ln \frac{H^{2}+r^{2}-R^{2}+\sqrt{r^{4}+2 r^{2}\left(H^{2}-R^{2}\right)+\left(H^{2}+R^{2}\right)^{2}}}{2 H^{2}}$, 
где $H$ и $R$ - расстояния рассматриваемой точки $K$ на рис. 2 соответственно от плоскости диска и от центра диска в его плоскости; $r=d / 2-$ радиус диска (остальные обозначения и их значения см. выше).

Преобразуем эту формулу в более удобный для нашего случая вид (при использовании этой формулы для разных дисков меняются только значения $H$ ):

$$
\dot{K}=\pi A_{s} \Gamma_{\delta} \ln \left[0,5\left(1+\frac{R^{2}-r^{2}}{H^{2}}\left(\sqrt{1+\frac{2\left(R^{2}+r^{2}\right)+H^{2}}{\left(R^{2}-r^{2}\right)^{2}} H^{2}}-1\right)\right] .\right.
$$

Учитывая примененную выше нумерацию дисков, имеем $H_{0}=0, H_{\mathrm{i}}=i l=0,22 i \mathrm{~cm}(i=1 \ldots 40)$. Подставив численные значения и учитывая, что $R=r+10=1,4+10=11,4 \mathrm{~cm}$, получим $\dot{W}=\dot{K}=6,5 \cdot 10^{6}$ а3в/с $=2,34 \cdot 10^{-2}$ мк3в/ч.

Как видим, это значение почти на два порядка меньше значений в требованиях [1]. Учитывая идентичность определяющих МЭД параметров радиаторов КД КНТ31 и КНT31-1 (активность, площадь радиатора, общая масса урана), с большой степенью уверенности можно предполагать, что значение МЭД, создаваемой КД КНТ31 в контрольной точке, имеет тот же порядок.

\section{Выводы}

Проведенный анализ позволяет сделать следующие заключения.

1. Все виды излучения, возникающие как при $\alpha$-распаде, так и при спонтанном делении имеющегося в КНТ31 и КНT31-1 урана (потоки нейтронов, $\gamma$-квантов, $\alpha-$ и $\beta$-частиц, а также осколки деления), не представляют радиационной опасности. Радиационная безопасность указанных излучений для рассматриваемых КД обусловлена крайне низкой активностью по отдельным видам излучений (нейтронному и $\beta$-излучению), а также пренебрежимо малыми значениями МЭД в контрольной точке (10 cм от поверхности КД) и расстояниями свободного пробега частиц (с учетом экранизации корпуса КД). Таким образом, все виды радиационных излучений, создаваемые материалом радиатора рассматриваемых КД, практически не оказывают радиационного воздействия на окружающую среду и обслуживающий персонал.
2. Необходимость проводить регистрацию ИИИ этих КД в Государственном регистре в соответствии с [1] отсутствует, так как одновременно не выполняются условия, перечисленные в П. 1 введения.

3. Масса ИИИ в одной КД $(0,5$ г) освобождает их от учета в [2], [3].

\section{Список литературы}

1. Порядок государственной регистрации источников ионизируюшего излучения. - УТв. Постановлением КМУ 16.11.2000 г. № 1718 .

2. НД-306-801-93. Государственная система учета и контроля ядерных материалов.

3. НП 306.7.122-206. Правила ведения учета и контроля ядерных материалов.

4. Дмитриев А. Б., Малышев Е. К. Нейтронные ионизационные камеры для реакторной техники. - М.: Атомиздат, 1975. - 94 с.

5. Малышев Е. К., Засадыи Ю. Б., Стабровский С. А.. Газоразрядные детекторы для контроля ядерных реакторов. - М.: Энергоатомиздат, 1991. - $158 \mathrm{c}$

6. Мухин K. Н. Введение в ядерную физику. - М.: Атомиздат, 1965. $-720 \mathrm{c}$

7. Абрамов А. И. Основы ядерной физики. - М.: Энергоатомиздат, 1983. $-256 \mathrm{c}$

8. Фролов В. В. Ядерно-физические методы контроля делящихся веществ. - М.: Энергоатомиздат, 1989. - 185 с.

9. Козлов В. Ф. Справочник по радиационной безопасности. М.: Энергоатомиздат, 1991. - 352 с.

10. Шаров Ю. Н., Шубин Н. В. Дозиметрия и радиационная опасность. - М.: Энергоиздат, 1982. - 256 с. 